\title{
AN INITIAL VALUE PROBLEM FOR A CLASS OF EQUATIONS OF MIXED TYPE ${ }^{1}$
}

\section{STEFAN BERGMAN}

1. Introduction. In connection with the consideration of second order linear partial differential equations of mixed type (that is, equations which are elliptic for some values of the independent variables and hyperbolic for others) there arises an initial value problem, namely that of expressing a solution of the equation in terms of the values of the solution and of its normal derivative on a portion of the boundary between the regions of elliptic and of hyperbolic behavior of the equation. Of particular interest are expressions in the form of single or multiple integrals representing the solution in terms of the prescribed data. These may enable us to deduce relationships between the initial data and various properties of the solution. ${ }^{2}$

In the present paper such a formula is derived for solutions of the following equation of mixed type:

$$
\begin{aligned}
\frac{\partial^{2} \psi}{\partial x^{2}}+(-x)^{s} \frac{\partial^{2} \psi}{\partial y^{2}} & =0 & & \text { for } x<0, \\
\frac{\partial^{2} \psi}{\partial x^{2}}-x^{s} \frac{\partial^{2} \psi}{\partial y^{2}} & =0 & & \text { for } x>0,
\end{aligned}
$$

where $s$ is any constant greater than -1 . For $s=1$ our equation coincides with one which has been studied in an important investigation by Tricomi [6], see also [5], and which is met in the theory of twodimensional flows of a compressible fluid when we investigate the streamfunction $\psi$ in the so-called hodograph plane and make a certain simplifying assumption concerning the equation of state of the fluid. See $[1,2,4]$. We note that the equation is elliptic for $x<0$, hyperbolic for $x>0$.

We shall derive here an integral representation for the solution of (1a), (1b) in terms of the prescribed values $\psi(0, y)=T(y)$ and

Received by the editors January 6, 1948, and, in revised form, February 11, 1948.

1 Paper done under Navy Contract NOrd 8555-Task F, at Harvard University. The ideas expressed in this paper represent the personal views of the author and not necessarily those of the Bureau of Ordnance.

${ }^{2}$ As has been found in the study of equations of elliptic type and those of hyperbolic type, it is possible to exploit various integral representations of their solutions for the investigation of their properties. For various possibilities in this direction see $\$ 3$ and $[3$, p. $140 \mathrm{ff}$.]. Numbers in brackets refer to the bibliography at the end of the paper. 
$\psi_{x}(0, y) \equiv(\partial \psi(x, y) / \partial x)_{x=0}=S(y)$, under the assumption that $T(y)$ and $S(y)$ are real analytic functions of $y$ and therefore can be analytically continued into a domain $B$ of the complex $\eta$-plane $\left(\eta=y+i y_{1}\right)$ which contains the portion of the real axis $\left(y_{1}=0\right)$ along which $T(y)$ and $S(y)$ are initially defined. The solution obtained will, for each fixed value of $y$, be analytic in the real variable $(-x)^{2+8}$ for $x<0$ and in the real variable $x^{2+s}$ for $x>0 .^{3}$

I should like to take this opportunity to thank A. Zeichner for his valuable assistance in connection with the present paper.

2. An integral representation for the solutions of equation (1). The solution $\psi$ will be obtained as the sum of two solutions, $\psi^{(1)}$ and $\psi^{(2)}$, of (1). These solutions will satisfy along the line $x=0$ the conditions:

$$
\begin{array}{ll}
\psi^{(1)}(0, y)=T(y), & \psi_{x}^{(1)}(0, y)=0, \\
\psi^{(2)}(0, y)=0, & \psi_{x}^{(2)}(0, y)=S(y) .
\end{array}
$$

Since (1) is linear and homogeneous, the desired solution $\psi$ may be obtained by superposition of $\psi^{(1)}$ and $\psi^{(2)}$.

THEOREM 1. Let

$$
\text { (4) }=\frac{1}{\pi^{2} i} \int_{t=0}^{\pi / 2} \int_{\mathrm{X}=0}^{1} \oint_{c} \frac{T(\eta)(1-\mathrm{X})^{-(2+s)^{-1}}\left[1+s-v^{2}(\eta-y)^{-2}\right]}{(2+s)(\eta-y)\left[1+v^{2}(\eta-y)^{-2}\right]^{2}} d t d \mathrm{X} d \eta
$$

where

$$
v=R X^{1 / 2} \sin t, \quad R \equiv R(x) \equiv(1+s / 2)^{-1}|x|^{1+s / 2}
$$

and where $C$ is a simple closed curve in the plane of the complex variable $\eta=y+i y_{1}$, which curve encloses the circle $|\eta-y|=R$. If $|x|$ is so small that the curve $C$ lies in the regularity domain of $T(\eta)$, then $\psi^{(1)}(x, y)$ represents a solution of (1a) which satisfies the initial conditions (2).

PROOF. In order to prove our assertions, let us first write the required solution in the form of a series:

$$
\begin{aligned}
\psi^{(1)}(x, y) & =T^{(0)}(y)+T^{(2)}(y)(-x)^{2+s}+T^{(4)}(y)(-x)^{2(2+s)}+\cdots \\
& =\sum_{n=0}^{\infty} T^{(2 n)}(y)(-x)^{n(2+s)} .
\end{aligned}
$$

${ }^{3}$ An interesting problem which we do not consider here is that of determining whether solutions of (1) exist which do not satisfy all the conditions of analyticity stated above. We note that here and in the following we write "(1)" instead of "(1a)" and "(1b)." 
Proceeding formally we obtain:

$$
(-x)^{s} \psi_{y y}^{(1)}=\sum_{n=0}^{\infty} T_{y y}^{(2 n)}(y)(-x)^{n(2+8)+8}
$$

$\psi_{x x}^{(1)}=\sum_{n=0}^{\infty} T^{(2 n+2)}(y)[(n+1)(2+s)][(n+1)(2+s)-1](-x)^{n(2+s)+s}$.

Adding these last two equations and taking account of (1a), we find that the functions $T^{(0)}, T^{(2)}, T^{(4)}, \cdots$ must satisfy

$$
T^{(2 n)}(y)=-\frac{T_{y y}^{(2 n-2)}(y)}{[n(2+s)][n(2+s)-1]}, \quad n=1,2,3, \cdots .
$$

By repeatedly applying this recursion formula, we can express $T^{(2 n)}(y)$ in terms of $d^{2 n} T(y) / d y^{2 n}$, namely:

$$
T^{(2 n)}(y)=\frac{(-1)^{n} d^{2 n} T(y) / d y^{2 n}}{\prod_{k=1}^{n}[k(2+s)][k(2+s)-1]}, \quad T^{(0)}(y) \equiv T(y) .
$$

Now let $C$ be a curve satisfying the conditions stated in the theorem. Then the Cauchy integral formula enables us to rewrite the above equation in the following manner:

$$
\begin{aligned}
2 \pi i T^{(2 n)}(y) & =\frac{(-1)^{n}(2 n) ! \oint_{c} \frac{T(\eta) d \eta}{(\eta-y)^{2 n+1}}}{\prod_{k=1}^{n}[k(2+s)][k(2+s)-1]} \\
& =\frac{(-1)^{n}(2 n) ! \oint_{c} \frac{T(\eta) d \eta}{(\eta-y)^{2 n+1}}}{n !(2+s)^{2 n} \prod_{k=1}^{n}\left[k-(2+s)^{-1}\right]}
\end{aligned}
$$

Now, by applying the well known identities: $\Gamma(n+1)=n !, \Gamma(2 n+1)$ $=(2 n) !$, and $p \Gamma(p)=\Gamma(p+1)$, we find that (6) may be rewritten

$$
\begin{aligned}
2 \pi i T^{(2 n)}(y)=\frac{(-1)^{n}\left[n+1-(2+s)^{-1}\right] \Gamma(n+1) \Gamma(2 n+1) \Gamma\left(1-(2+s)^{-1}\right)}{(2+s)^{2 n}[\Gamma(n+1)]^{2} \Gamma\left(n+2-(2+s)^{-1}\right)} \\
\times \oint_{c} \frac{T(\eta) d \eta}{(\eta-y)^{2 n+1}} .
\end{aligned}
$$


Now, since

$$
\begin{gathered}
\frac{\Gamma(2 n+1)}{[\Gamma(n+1)]^{2}}=\frac{2^{2 n+1}}{\pi} \int_{0}^{\pi / 2} \sin ^{2 n} t d t \\
\frac{\Gamma(n+1) \Gamma\left(1-(2+s)^{-1}\right)}{\Gamma\left(n+2-(2+s)^{-1}\right)}=\int_{0}^{1} \mathrm{X}^{n}(1-\mathrm{X})^{-(2+s)} d \mathrm{X},
\end{gathered}
$$

we obtain finally:

$$
2 \pi^{2} i T^{(2 n)}(y)=(-1)^{n}\left[n+1-(2+s)^{-1}\right](2+s)^{-2 n} 2^{2 n+1}
$$

$$
\times \int_{t=0}^{\pi / 2} \sin ^{2 n} t d t \int_{\mathrm{X}=0}^{1} \mathrm{X}^{n}(1-\mathrm{X})^{-(2+s)^{-1}} d \mathrm{X} \oint_{c} \frac{T(\eta) d \eta}{(\eta-y)^{2 n+1}}
$$

If we multiply both sides of $(8)$ by $(-x)^{n(2+8)}$ and sum for $n=0,1,2, \cdots$, we obtain:

$$
\begin{aligned}
2 \pi^{2} i \psi^{(1)}(x, y)=\sum_{n=0}^{\infty} \int_{t=0}^{\pi / 2} & \int_{\mathrm{X}=0}^{1} \oint_{c} \frac{2 T(\eta)(1-\mathrm{X})^{-(2+s)^{-1}}}{(\eta-y)} \\
& \times\left[n+1-(2+s)^{-1}\right]\left\{\frac{-v^{2}}{(\eta-y)^{2}}\right\}^{n} d t d \mathrm{X} d \eta
\end{aligned}
$$

Let the minimum distance of the point $y$, considered temporarily fixed, from the curve $C$ be $\geqq R+\delta$ (see (5)), $\delta$ any small positive constant. Then, since $\mathrm{X}$ and $\sin ^{2} t$ never exceed one in absolute value,

$$
\sum_{n=0}^{\infty}\left[n+1-(2+s)^{-1}\right]\left[\frac{-4 \mathrm{X}(-x)^{2+s} \sin ^{2} t}{(2+s)^{2}(\eta-y)^{2}}\right]^{n}
$$

will converge absolutely and uniformly since $4(-x)^{2+s} /(2+s)^{2}|\eta-y|^{2}$ $\leqq(R /(R+\delta))^{2} \leqq 1-\epsilon$, and the sum of the above series will be $(u+1+s) /(2+s)(1-u)^{2}$ where $u=-4 \mathrm{X}(-x)^{2+s} \sin ^{2} t /(2+s)^{2}(\eta-y)^{2}$ $=-v^{2} /(\eta-y)^{2}$. Since we may interchange the order of summation and integration, we thus obtain from (9):

$$
\text { (10) }=\frac{1}{\pi^{2} i} \int_{t=0}^{\pi / 2} \int_{\mathrm{x}=0}^{1} \oint_{C} \frac{T(\eta)(1-\mathrm{X})^{-(2+s)^{-1}}\left[1+s-v^{2}(\eta-y)^{-2}\right]}{(2+s)(\eta-y)\left[1+v^{2}(\eta-y)^{-2}\right]^{2}} d t d \mathrm{X} d \eta \text {. }
$$

\section{COROLlaRY 1. The function}

$$
\psi^{(1)}(x, y)
$$

$$
=\frac{1}{\pi^{2} i} \int_{t=0}^{\pi / 2} \int_{\mathrm{X}=0}^{1} \oint_{C} \frac{T(\eta)(1-\mathrm{X})^{-(2+s)^{-1}}\left[1+s+v^{2}(\eta-y)^{-2}\right]}{(2+s)(\eta-y)\left[1-v^{2}(\eta-y)^{-2}\right]^{2}} d t d \mathrm{X} d \eta
$$


represents, for sufficiently small positive values of $x, a$ solution of (1b) which satisfies the initial conditions (2).

Proof. The derivation of formula (11) is entirely parallel to that of (4). We have only to take into account that $(-x)^{8}$ has to be replaced by $-x^{8}$.

THEOREM 2. The expression

$$
\begin{aligned}
& \psi^{(2)}(x, y) \\
& \text { (12) }=\frac{x}{\pi^{2} i} \int_{t=0}^{\pi / 2} \int_{\mathrm{X}=0}^{1} \oint_{C} \frac{S(\eta)(1-\mathrm{X})^{(2+s)^{-1}\left[3+s+v^{2}(\eta-y)^{-2}\right]}}{(2+s)(\eta-y)\left[1+v^{2}(\eta-y)^{-2}\right]^{2}} d t d \mathrm{X} d \eta \text {, }
\end{aligned}
$$

where $C$ and $\eta$ have the meaning explained in Theorem 1, and $S(y)$ is an arbitrary real analytic function of one real variable, represents a solution of (1a) which satisfies the initial conditions (3).

PROof. The proof proceeds exactly as in Theorem 1. We write:

$$
\psi^{(2)}(x, y)=-\sum_{n=0}^{\infty} T^{(2 n+1)}(y)(-x)^{1+n(2+s)}, \quad T^{(1)}(y) \equiv S(y),
$$

which formally satisfies conditions (3). Proceeding as in the proof of Theorem 1, we find that the $T^{(2 n+1)}$ can be expressed as derivatives of $T^{(1)}$, namely:

$$
T^{(2 n+1)}(y)=\frac{(-1)^{n} d^{2 n} T^{(1)}(y) / d y^{2 n}}{n !(2+s)^{2 n} \prod_{k=1}^{n}\left(k+(s+2)^{-1}\right)}, \quad n=1,2,3, \cdots
$$

or:

$$
\begin{aligned}
-2 \pi^{2} i T^{(2 n+1)}(y)(-x)^{1+n(2+s)} & \\
= & x \frac{2^{2 n+1}\left[n+1+(2+s)^{-1}\right](-1)^{n}(-x)^{n(s+2)}}{(2+s)^{2 n}} \\
& \cdot \int_{t=0}^{\pi / 2} \int_{\mathrm{X}_{=0}}^{1} \oint_{C} \frac{S(\eta)}{(\eta-y)^{2 n+1}} \cdot \sin ^{2 n} t \cdot \mathrm{X}^{n}(1-\mathrm{X})^{(s+2)^{-1}} d t d \mathrm{X} d \eta
\end{aligned}
$$

Summing for $n=0,1,2,3, \cdots$, we get, for values of $x$ sufficiently close to zero:

$$
\begin{aligned}
& 2 \pi^{2} i \psi^{(1)}(x, y) \\
& =-x \int_{t=0}^{\pi / 2} \int_{\mathrm{X}=0}^{1} \oint_{c} \frac{2 S(\eta)(1-\mathrm{X})^{(2+s)^{-1}\left[3+s+v^{2}(\eta-y)^{-2}\right]}}{(2+s)(\eta-y)\left[1+v^{2}(\eta-y)^{-2}\right]^{2}} d t d \mathrm{X} d \eta
\end{aligned}
$$


which yields the statement of Theorem 2 .

Finally, by repeating the reasoning used in the proof of Corollary 1 , we obtain:

Corollary 2. The expression

$$
\begin{aligned}
& \psi^{(2)}(x, y) \\
& =\frac{x}{\pi^{2} i} \int_{t=0}^{\pi / 2} \int_{\mathrm{X}=0}^{1} \oint_{C} \frac{S(\eta)(1-\mathrm{X})^{(2+s)^{-1}}\left[3+s-v^{2}(\eta-y)^{-2}\right]}{(2+s)(\eta-y)\left[1-v^{2}(\eta-y)^{-2}\right]^{2}} d t d \mathrm{X} d \eta
\end{aligned}
$$

represents, for sufficiently small positive values of $x$, a solution of (1b) which satisfies the initial conditions (3).

3. Concluding remarks. In $\$ 2$ we did not indicate explicitly the domain of the $x y$-plane in which the representations (4), (11), (12), (17) hold, but an almost obvious consideration shows that representations (4) and (11) are valid in the domain ${ }^{4} \sum_{Y \in J}[y=Y, R(x)<r(Y)]$ (see (5)), where $J$ denotes the intersection of the (real) $y$-axis with the regularity domain $B$ of $T(\eta), \eta=y+i y_{1}$, in the (complex) $\eta$-plane and $r(Y)$ the distance of $Y$ from the boundary of $B$. (It should be mentioned that using, in addition, certain summation methods, one might obtain representations which are valid in larger domains of the $x y$-plane.)

If $T(y)$ and $S(y)$ become, upon continuation into the complex $\eta$-plane, entire functions, then the above representation can be used to define $\psi(x, y)$ for all values of $x$ and $y$, for in this case the curve $C$ can always be chosen in such a way as to provide the determination $\psi(x, y)$ at any preassigned point. ${ }^{5}$

As has been shown in another place, integral formulas transforming analytic functions of one complex variable into solutions of a linear partial differential equation can be used in order to derive various results from theorems in the theory of functions pertaining to solutions of these equations. As an example for such a procedure, we shall obtain upper bounds for the growth of $\psi^{(1)}$ and $\psi^{(2)}$ from the derivatives, $n ! a_{n}$ of $T(y)$ and $n ! b_{n}$ of $S(y)$ respectively, at some fixed point, say at the origin.

In order to obtain this relation, we shall rewrite the formulas (4), (11), (12), and (17) in a certain modified form, which form holds in the case where $T(\eta)$ or $S(\eta)$ are entire functions.

- $\sum_{Y \in J}$ denotes the sum of all points $x, y$ where for every $Y, x$ ranges over the interval indicated in the bracket, and $Y$ ranges over $J$.

${ }^{5}$ In order to apply the method of $\$ 2$ in the case where $T(y)$ and $S(y)$ are not analytic functions of one real variable $y$, we have to approximate $T(y)$ and $S(y)$ by analytic functions, say by polynomials. 
LEMMA 3.1. Under the assumption that $T(\eta)$ is an entire function of a complex variable, the representation (4) can be written in the form

$$
\begin{aligned}
\psi^{(1)}(x, y)=\frac{2}{\pi} \mathrm{B}\left(\frac{1}{2}\right. & \left., \frac{1+s}{2+s}\right) \int_{q=0}^{1}\left[\frac{1+s}{2+s}\{\operatorname{Re} T(y+i R q)\}\right. \\
& \left.-\frac{R q}{2}\left\{\operatorname{Im} T^{\prime}(y+i R q)\right\}\right]\left(1-q^{2}\right)^{s /(4+2 s)} d q
\end{aligned}
$$

$(\operatorname{Re}=$ Real part of $\cdots, \operatorname{Im}=$ Imaginary part of $\cdots)$.

Proof. The proof of representation (18) follows immediately by the use of the Cauchy integral formula. We may write (4) in the form

$$
\begin{aligned}
\psi^{(1)}(x, y) & =\frac{1}{\pi^{2} i} \int_{t=0}^{\pi / 2} \int_{\mathrm{X}=0}^{1} \\
& \cdot\left\{\oint_{c} \frac{T(\eta)(\eta-y)\left[(1+s)(\eta-y)^{2}-v^{2}\right] d \eta}{(2+s)\left[(\eta-y)^{2}+v^{2}\right]^{2}}\right\}(1-\mathrm{X})^{-(2+s)^{-1}} d t d \mathrm{X} .
\end{aligned}
$$

Since we assume that $x<0, v$ can vanish only when $\mathrm{X}=0$, or $t=0$.

On the other hand, it is easy to show that (19) may also be written in the form

$$
\begin{aligned}
& \psi^{(1)}(x, y)=\lim _{\substack{\rightarrow \rightarrow 0, \delta \rightarrow 0 \\
\pi^{2} i}} \int_{t=\epsilon}^{\pi / 2} \int_{\mathrm{X}=\delta}^{1} \\
& \times\left\{\oint_{C} \frac{T(\eta)(\eta-y)\left[(1+s)(\eta-y)^{2}-v^{2}\right] d \eta}{(2+s)\left[(\eta-y)^{2}+v^{2}\right]^{2}}\right\}(1-\mathrm{X})^{-(2+s)^{-1}} d t d \mathrm{X} .
\end{aligned}
$$

In order to evaluate the integral in the braces, we now apply the Cauchy theorem.

Since $v$ never becomes 0 , the only poles of the integrand are $\eta=y \pm i v$. In order to determine the value of the integral in the braces, we have to determine the residues of the integrand considered as a function of $\eta$ at the points $\eta=y \pm i v$. A formal computation yields

$$
\begin{aligned}
& \frac{T(\eta)(\eta-y)\left[(1+s)(\eta-y)^{2}-v^{2}\right]}{(2+s)\left[(\eta-y)^{2}+v^{2}\right]^{2}} \\
& =\left(\frac{1+s}{2+s}\right) \frac{1}{2}\left[\frac{T(\eta)}{\eta-y+i v}+\frac{T(\eta)}{\eta-y-i v}\right] \\
& \quad-\frac{i v}{4}\left[\frac{T(\eta)}{(\eta-y+i v)^{2}}-\frac{T(\eta)}{(\eta-y-i v)^{2}}\right] .
\end{aligned}
$$

Using (20), (21) and Cauchy's integral formula, we obtain 


$$
\begin{aligned}
\psi^{(1)}(x, y)=\frac{2}{\pi} \int_{t=0}^{\pi / 2} & \int_{\mathrm{X}=0}^{1}\left[\frac{1+s}{2+s}\{\operatorname{Re} T(y+i v)\}\right. \\
& \left.-\frac{v}{2}\left\{\operatorname{Im} T^{\prime}(y+i v)\right\}\right](1-\mathrm{X})^{-(2+s)^{-1}} d t d \mathrm{X}
\end{aligned}
$$

Equation (22) may also be written in the form

$$
\begin{aligned}
\psi^{(1)}(x, y)=\lim _{\delta \rightarrow 0, \epsilon \rightarrow 0} \frac{2}{\pi} \int_{t=0}^{(\pi / 2)-\delta} \int_{\mathrm{X}=0}^{1-\epsilon}\left[\frac{1+s}{2+s} \operatorname{Re} T(y+i v)\right. \\
\left.-\frac{v}{2} \operatorname{Im} T^{\prime}(y+i v)\right](1-\mathrm{X})^{-(2+s)^{-1}} d t d \mathrm{X} .
\end{aligned}
$$

If now we introduce the variables [see (5)]

$$
q=\mathrm{X}^{1 / 2} \sin t \equiv \frac{v}{R}, \quad \tau=\frac{\mathrm{X}^{1 / 2} \cos t}{\left[1-\mathrm{X} \sin ^{2} t\right]^{1 / 2}}
$$

that is,

$$
\mathrm{X}=\tau^{2}+q^{2}-\tau^{2} q^{2}, \quad t=\arctan \left[q \tau^{-1}\left(1-q^{2}\right)^{-1 / 2}\right]
$$

and pass to the limit, we obtain the representation

$$
\begin{aligned}
& \psi^{(1)}(x, y)=\frac{4}{\pi} \int_{q=0}^{1} \int_{\tau=0}^{1}\left[\frac{1+s}{2+s} \operatorname{Re} T(y+i R q)\right. \\
& \left.-\frac{R q}{2} \operatorname{Im} T^{\prime}(y+i R q)\right]\left(1-q^{2}\right)^{s /(4+2 s)}\left(1-\tau^{2}\right)^{-(2+s)^{-1}} d \tau d q .
\end{aligned}
$$

Integration of the right-hand side with respect to $\tau$ yields the desired result.

Exactly the same considerations can be repeated in the case of representations (11), (12), and (17).

THEOREM 3.1. Let $\psi^{(1)}$ be an analytic solution of (1a) satisfying condition (2). If $T(y)=\sum_{n=0}^{\infty} a_{n} y^{n}$ is an entire function (when continued to the complex values of the argument), then $\psi^{(1)}(x, y)$ can be extended to all values of $y$ and $x, x<0 . \psi^{(1)}$ satisfies the inequality

$$
\begin{gathered}
\left|\psi^{(1)}(x, y)\right| \leqq M e^{\sigma r^{\rho}}\left[1+2(1+s)^{-1}(-x)^{(2+s) / 2}\right], \\
r=\left[y^{2}+4(2+s)^{-2}(-x)^{2+s}\right]^{1 / 2}
\end{gathered}
$$

where $M$ is a suitably chosen constant and $\rho$ and $\sigma$ are given by

$$
\rho=\limsup _{n \rightarrow \infty} \frac{n \log n}{\log \left(1 /\left|a_{n}\right|\right)}, \quad(\sigma \rho e)^{1 / \rho}=\limsup _{n \rightarrow \infty} n^{1 / \rho}\left(\left|a_{n}\right|\right)^{1 / n} .
$$


The proof of this inequality follows immediately from the representation (18) and the fact that by classical results of the theory of entire functions of one complex variable, the inequality $|T(\eta)|$ $\leqq M e^{\sigma|\eta|^{\rho}}$ holds.

The expression (18) can be simplified further when $s \geqq 0$ by using the fact that $-R\left[\operatorname{Im} T^{\prime}(y+i R q)\right]=d[\operatorname{Re} T(y+i R q)] / d q$ and then integrating by parts. This yields:

$$
\psi^{(1)}(x, y)=\frac{2}{\pi} \mathrm{B}\left(\frac{1}{2}, \frac{1+s}{2+s}\right) \frac{s}{4+2 s}
$$

$$
\begin{array}{rlr}
\times \int_{q=0}^{1}[\operatorname{Re} T(y+i R q)]\left(1-q^{2}\right)^{(-4-s) /(4+2 s)} d q & \text { for } s>0, \\
\psi^{(1)}(x, y)=\operatorname{Re}[T(y+i x)] & \text { for } s=0 .
\end{array}
$$

By noting the fact that

$$
\begin{aligned}
\int_{0}^{1}\left(1-q^{2}\right)^{-(4+s) /(4+2 s)} d q & =\frac{1}{2} \mathrm{~B}\left(\frac{1}{2}, \frac{s}{4+2 s}\right) \\
& =\left[\frac{2}{\pi} \mathrm{B}\left(\frac{1}{2}, \frac{1+s}{2+s}\right) \frac{s}{4+2 s}\right]^{-1}
\end{aligned}
$$

we finally obtain the formula

$$
\psi^{(1)}(x, y)=\frac{\int_{q=0}^{1}[\operatorname{Re} T(y+i R q)]\left(1-q^{2}\right)^{(-4-s) /(4+2 s)} d q}{\int_{q=0}^{1}\left(1-q^{2}\right)^{(-4-s) /(4+2 s)} d q} .
$$

Thus for $s \geqq 0$, we obtain the following result.

THEOREM 3.2. Let $\psi^{(1)}$ be an analytic solution of (1a) satisfying condition (2). If $T(\eta)$ is entire and $s \geqq 0$, then using the notation of Theorem 3.1 , we obtain

$$
\left|\psi^{(1)}(x, y)\right| \leqq M e^{\sigma r^{p}}
$$

The proof of this inequality follows immediately from (28) and (29).

\section{BIBLIOGRAPHY}

1. Stefan Bergman, Operator methods in the theory of compressible fluids, Proceedings of the 1st Symposium for Applied Mathematics, Publication of the American Mathematical Society, 1949.

2. - Two-dimensional subsonic flows of a compressible fluid and their singularities, Trans. Amer. Math. Soc. vol. 61 (1947) pp. 452-498. 
3. - Linear operators in the theory of partial differential equations, Trans. Amer. Math. Soc. vol. 53 (1943) pp. 130-155.

4. ——, Two dimensional transonic flow patterns, Amer. J. Math. vol. 70 (1948) pp. 956-891.

5. E. Holmgren, Sur un problème aux limites pour l'equation $y^{m} \partial^{2} z / \partial x^{2}+\partial^{2} z / \partial y^{2}=0$, Arkiv for Mathematik, Astronomi och Fysik vol. 19, B (1927) pp. 1-3.

6. F. Tricomi, Sulle equazioni lineari alle derivate parzialo di $2^{\circ}$ ordine di tipo misto, Memorie della R. Accademia dei Lincei vol. 14 (1923) pp. 133-247.

HARVARD UNIVERSITY

\section{AN INVERSION FORMULA FOR THE GENERALIZED STIELTJES TRANSFORM}

D. B. SUMNER ${ }^{1}$

1. Introduction. The problem of finding formulae to invert the Stieltjes transforms

$$
\begin{aligned}
& f(x)=\int_{0}^{\infty} d \alpha(t) /(x+t), \\
& f(x)=\int_{0}^{\infty} \phi(t) d t /(x+t),
\end{aligned}
$$

and the generalized transforms

$$
\begin{aligned}
& f(x)=\int_{0}^{\infty} d \alpha(t) /(x+t)^{\rho}, \\
& f(x)=\int_{0}^{\infty} \phi(t) d t /(x+t)^{\rho},
\end{aligned}
$$

has been solved by Widder [A, pp. 7-60 $]^{2}$ and by Pollard [F, pp. 1416]. The function $\phi(t) \in L(0, \infty), \alpha(t)$ is a normalized function of

Received by the editors January 19, 1948, and, in revised form, February 26, 1948.

1 The subject matter of this paper forms part of a dissertation presented to the University of the Witwatersrand. The author wishes to acknowledge with gratitude facilities granted by the Principal and Council of that University, and assistance subsequently given by the Council for Scientific and Industrial Research of the Union of South Africa. In particular, the author acknowledges his indebtedness to Dr. J. P. Dalton, Emeritus Professor in Mathematics of the University of the Witwatersrand.

2 Roman letters in brackets refer to the bibliography at the end of the paper. 